\section{MS34-P16 Structural chemistry of} piperidine hydrates

Paweł Socha ${ }^{1}$, Łukasz Dobrzycki ${ }^{1}$, Michał K. Cyrański ${ }^{1}$, Roland Boese $^{1}$

1. Czochralski Laboratory of Advanced Crystal Engineering, Faculty of Chemistry, University of Warsaw, Żwirki i Wigury 101, 02-093 Warsaw, Poland

email: psocha@chem.uw.edu.pl

The aim of this report was to investigate the possibility of creating hydrates or clathrates for piperidine-water system. Piperidine is a heterocyclic aliphatic amine with six-membered ring. Crystal structure of the amine is known, molecules build columns in crystal lattice [1]. Hydrates were crystalized on the diffractometer, using in situ crystallisation technique with IR laser [2]. Five hydrates have been received during the project, consisting of $0.50,2.00,8.10,9.75$ and 11.00 water per one amine molecule. Structures of hydrates were determined with single crystal X-ray diffraction. Interestingly, hydrates with high concentration of water were very similar to gas clatrathates [3], however there were hydrogen bonds between amine and water in crystal lattice and the water framework including positions of $\mathrm{H}_{2} \mathrm{O}$ molecules was disordered. What is more, piperidine hydrates consisting 9.75 and 11.00 water were isostructural with an analogue structure of tert-butylamine hydrates [4, 5]. Packing diagrams of these two structures are presented in the Figure below. Moreover, piperidine hydrate 8.10 were isostructural with iso-propylamine hydrate 8.00 [6, 7]. All synthesised crystals were also characterised with Raman spectroscopy and X-ray powder diffraction.

Acknowledgements The work has been supported by the National Science Center grant (NCN 2011/03/B/ST4/02591).

\section{References}

[1] Parkin, A., Oswald, I. D. H., Parsons S. (2004). Acta Crystallogr. B., 60, 219.

[2] Boese, R. (2014). Z. Kristallogr. 229, 595.

[3] Sloan, E. D. "Clathrate Hydrates of Natural Gases", Marcel Dekker, New York, USA, 1990.

[4] McMullan, R. K., Jeffrey, G. A., Jordan, T. H. (1967). J. Chem. Phys., 47, 1229; Jeffrey, G. A. (1969). Acc. Chem. Res., 11, 344.

[5] Dobrzycki, Ł., Taraszewska, P., Boese, R., Cyrański, M. K., Cirkel, S.A. (2015). Angewandte Chemie International Edition, 54, 10138.

[6] McMullan, R. K., Jeffrey, G. A., Panke, D. (1970). J. Chem. Phys., 53, 3568.

[7] McMullan, R. K., Jordan, T. H., Jeffrey, G. A. (1967) J. Chem. Phys., 47, 1218.

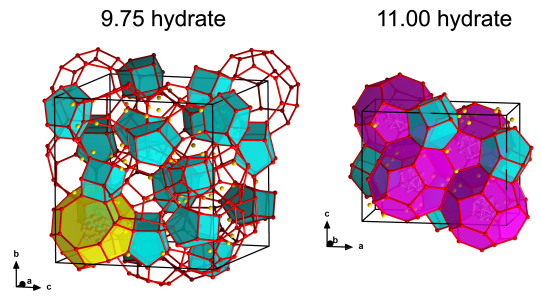

Figure 1. Unit cell content of 9.75 and 11.00 hydrates of piperidine. Alternative positions of $\mathrm{H}_{2} \mathrm{O}$ molecules indicating disorder of water framework are presented as yellow spheres.

Keywords: Hydrates, Clathrahes, Piperidine, in situ crystallization, Crystal Engineering, Single Crystal Diffraction, Raman Spectroscopy 